Abdelahad Chraibi*, Said Kharraja, Ibrahim H. Osman and Omar Elbeqqali

\title{
A Multi-Agents System for Solving Facility Layout Problem: Application to Operating Theater
}

https://doi.org/10.1515/jisys-2017-0081

Received March 4, 2017; previously published online September 5, 2017.

\begin{abstract}
Facility layout problem (FLP) has a great impact on the efficiency of any organization. It is concerned with defining the optimal location for each facility in order to optimize the supply chain productivity. In this kind of problems, the choice of resolution approach depends on the complexity and the size of the problem. Operating theaters are generally big structures containing a lot of facilities, which makes the conception of their layout a complex problem. In the literature, exact methods are powerless when faced with problem sizes up to 18 facilities. This leads us to explore other approaches, looking for efficient solutions. This paper presents a novel approach using a multi-agents system where agents' skills are exploited to cover a wide research space, to accelerate the decision-making process and to deal with real-life problem sizes. This decision-making tool is based on several mixed integer linear programming models for solving the FLP, and considers two types of environments with deterministic and variant patient demand. Several experiments have been performed to demonstrate the effectiveness of our approach, and several problem instances with $>80$ facilities have been solved in reasonable time.
\end{abstract}

Keywords: Static facility layout problem, adaptive facility layout problem, optimization, multi-agents system, healthcare logistics, mixed integer linear programming.

\section{Introduction}

The facility layout problem (FLP) is a common industrial problem of allocating facilities with the objective of either maximizing the adjacency requirement, minimizing the traveling costs, minimizing the building costs or minimizing the rearrangement costs. The objectives can be multiple; however, in hospitals, the objectives are patient oriented to ensure their satisfaction and security. The workplace structure is one of the important aspects in optimization processes to provide services with high quality for patients at a lower cost while maintaining the well-being of staff. In fact, a good placement of facilities contributes, in general, to the overall efficiency of operations and can reduce the total operating expenses up to 50\% [30].

The conception of an operating theater (OT) is directly influenced not only by the evolution of used materials and technologies, the continuing growth of population demand for care services, and the emergence of new diseases or other health risks, but also by economical, social and political factors. Nowadays, the OT layout is designed through traditional methods based on benchmarks and professional experiences, without using intelligent decision-making tools. These traditional methods proved to be inefficient due to the absence

\footnotetext{
*Corresponding author: Abdelahad Chraibi, University of Valenciennes and Hainaut-Cambresis, LAMIH UMR CNRS 8201, Valenciennes, France, e-mail: abdelahad.chraibi@univ-valenciennes.fr. https://orcid.org/0000-0003-2894-5594

Said Kharraja: University of Lyon, University of Saint-Etienne, Laboratory of Signal and Industrial Process Analysis (LASPI), Roanne, France Ibrahim H. Osman: Suliman S. Olayan's School of Business, Business Information and Decision Systems, American University of Beirut, Beirut, Lebanon

Omar Elbeqqali: Faculty of Science Dhar El Mahraz, University Sidi Mohamed Ben Abdellah, Laboratory of Informatics Image Processing and Numerical Analysis (LIIAN), Fez, Morocco
} 
of an optimization aspect and the high cost of maintenance [8]. The actual technological advancements invite hospitals to invest in these intelligent solutions in order to optimize their process and to increase efficiency and performance.

Aware of this need, we proposed, based on past several approaches using exact resolution methods, to deal with a small-sized OT layout problem (OTLP) [3]. This paper considers these results and build on to offer sufficient solutions for large-sized problems by using a multi-agents (MA) system (MAS). Today, the interest in using and implementing MAS no longer needs to be demonstrated. The research on complex agentoriented systems has a wide variety of industrial, business, science and educational applications. The design of these systems requires adequate methodologies to cope with agent-specific characteristics, which result from an agent-oriented domain analysis.

In a MAS, agents communicate with each other to share results or request resources, which greatly increases the complexity of the system. This is why the role of the coordinator agent is important to ensure the smooth interaction and running of the system. The communication between agents can take two different forms: (a) the negotiation protocol resulting from a competition between agents on a common objective or (b) the planning protocol resulting from the cooperation between agents, each one having a task of the global objective [24].

The major contributions of this work can be described in three points: (a) being among the first studies to use a MAS with two negotiation and cooperation protocols to solve such a combinatory problem - in fact, there is one paper dealing with the quadratic assignment problem, which is a relaxed version of the FLP; (b) the use a multi-objective resolution by considering both qualitative and quantitative aspects to minimize costs and maximize the satisfaction of security and hygiene norms while solving the OTLP - many works in the literature are dealing with the hospital layout problem, but all use theoretical graph methods that do not consider cost minimization; (c) the consideration of the two possible scenarios of an OT with a static environment in which patient demand is supposed to be constant for the whole planning horizon, and an uncertain environment that supposes that patient demand is not stable from a period to another in the planning horizon and that the facilities should be adaptive to demand change requirements. All considered constraints are retained from international norms and standards governing the healthcare facilities design and construction (Facility Guideline Institute, Guidelines for Design and Construction of Health Care Facilities).

This paper is organized as follows: Section 2 presents a literature review of FLP works in general and works using MAS to solve different industrial problems. Sections 3 and 4 contain the developed models using mixed integer linear programming (MILP) and MAS for the static OTLP (SOTLP) and adaptive OTLP (AOTLP), respectively. Experiments and results are shown in Section 5. Finally, we conclude and give a description of future research perspectives in Section 6.

\section{Literature Review}

In this section, we present first an overall FLP review and we conclude with works related to healthcare and specifically OT.

In the static FLP (SFLP), it is assumed that there is a deterministic flow of products/people between facilities over a single period planning horizon. SFLP has been solved using different solution approaches classified as either exact or approximate methods.

Hathhorn et al. [11] developed a multi-objective mixed integer programming (MIP) model for multi-floor FLP to minimize both the material handling cost and the total facility building cost, and used a lexicographic ordering technique to handle the multiple objectives. Zhang and Che [33] presented a new formulation of FLP using a MILP formulation to assign a certain number of rooms to a given number of departments while maximizing the utilization rate of the rooms. Deechongkit and Srinon [6] developed a MIP model for FLP without loss of department area by ignoring the lower and upper limits on the length/width of departments. Tari and Neghabi [28] presented a new linear adjacency approach for unequal area FLP and presented a MIP model to maximize the total adjacency rating among rectangular departments. Hammad et al. [10] developed 
a multi-objective MIP model for construction site layout planning to minimize noise pollution and transport costs.

Lin et al. [19] proposed a qualitative approach to design and optimize OTFL in hospitals. First, a systematic layout planning (SLP) is applied to design OTFL and they applied fuzzy constraint theory to evaluate the layout schemes. To address the unequal area multi-objective FLP, Ripon et al. [26] used a variable neighborhood search (VNS) with an adaptive scheme to present the final layouts as a set of Pareto-optimal solutions. Lorenz et al. [20] presented an adjacency-based method to hospital layout planning using a Newtonian gravitation model to propagate changes to a single relationship immediately to the whole space layout.

Kothari and Ghosh [15] presented four scatter search algorithms to solve large-sized single-row FLP. Each of these algorithms use a diversification generation method to generate initial populations, an improvement method to improve the permutations in the algorithm, a reference set update method to build and maintain a reference set of good quality permutations, and a solution combination method to update the reference set with new permutations. Kothari and Ghosh [14] presented a genetic algorithm (GA) to solve the singlerow FLP where all the individuals in a generation are subjected periodically to local search to improve their fitness. Lenin et al. [18] used a tabu search for multi-objective single-row FLP to find the linear machine sequence while minimizing the total investment cost of machines, the total material handling cost, the total number of machines in the final sequence, and the total flow distance of the products in units. Shivhare and Bansal [27] used a particle swarm optimization (PSO) algorithm to address the bidirectional loop FLP in a flexible manufacturing system with the objective to minimize the distance traveled by automated guided vehicles and the number of backtracking. Palubeckis [25] used a fast simulated annealing (SA) for single-row equidistant facility layout. The proposed algorithm provides a possibility to employ either merely pairwise interchanges of facilities or merely insertion moves, or both of them, and incorporates an innovative method for computing gains of both types of moves: pairwise interchanges and insertions.

The dynamic FLP (DFLP) is concerned with the design of multi-period facility layout plans and assumes a variable flow of products/people between facilities in each period of the planning horizon. DFLP has also been solved using different approaches classified as either exact or approximate methods.

Huang and Wong [12] developed a binary MILP to deal with site layout problem with multiple construction stages, and solved it using a standard branch-and-bound algorithm. The objective function was to minimize the total cost and relocation costs for all of the involved site facilities in each construction stage, and the model included safety constraints that were expressed by simple linear constraint sets.

Nahas and Nourelfath [22] proposed an iterated great deluge for the discrete dynamic FLP composed of two main steps. In the first step, a local optimum solution is generated using an extended great deluge algorithm. In the second step, the best current solution is perturbed and another extended great deluge algorithm is applied to improve the just ruined solution.

Chen [2] presented two binary coded hybrid ant colony optimizations for large DFLP. These methods used encoding and decoding schemes for solution representation to improve the swapping rearrangement of facilities within their meta-heuristic framework. Uddin [31] introduced a hybrid GA and VNS for dynamic FLP. To ensure a strong cooperation of GA and VNS, the population was split into two parts, where each part was assigned to a method considering a hybridizing coefficient to adjust the percentage of splitting population. Kulturel-Konak and Konak [17] used a large-scale hybrid SA algorithm for dynamic FLP, while the assumption of fixed department dimensions has been relaxed using the polyhedral outer approximation method to model non-linear constraints for the minimum department area requirements. The proposed approach was shown to be effective and versatile as it can be applied to various FLPs. Asl and Wong [1] investigated the unequal area DFLP and suggested a modified PSO in order to minimize the sum of the material handling costs and sum of the rearrangement costs. They used departments and periods swapping operators to improve the solutions. Two local search methods were used to find the best solution.

MAS has been developed in the context of distributed artificial intelligence and consists of a set of distributed cooperating agents, each of which acts autonomously. It provides an alternative approach to complex problems in a distributed manner where decisions should be based on processing of information from various sources of diverse nature [32]. 
MAS is used in different domains. For FLP, Tarkesh et al. [29] were the only ones to present an algorithm using a society of virtual intelligent agents to efficiently solve this problem, introducing thereby the concepts of emotion for the first time in operational research. They modeled each agent as a facility having proprieties such as money and emotions, which are adjusted during the agents' interactions, defined by a market mechanism in which the richer agents can pay extra money to obtain better locations and are less interested in lower ones. Nataraj Urs et al. [23] presented a distributed MA framework for the very-large-scale integration layout design process. They divided the entire floor plan task into many subtasks, and affected each one to an agent to solve it. Finally, they made agents interact with each other to negotiate the final placement for the design process.

Cossentino et al. [5] presented an MA simulation tool for decision making in automatic warehouse management. Their proposed a MAS aiming to optimize the suitable number of automated guided vehicles used for unloading containers that arrive in the warehouse. Nfaoui et al. [24] developed an agent-based distributed architecture for collaborative decision-making processes within the global distribution supply chain for best management of the unexpected rush order for which the quantity of products cannot be delivered partially or completely from the available inventory.

In healthcare, Isern et al. [13] presented a review of the literature about applications of agents regrouping $>15$ works and classified each one according to the main goal of the systems. González-Vélez et al. [9] presented an agent-based distributed decision support system for the diagnosis and prognosis of brain tumors developed by the HEALTHAGENTS project. Koutkias et al. [16] presented an interactive MAS for the management of patients' chronic diseases with the objective of detecting anomalous cases, and informed personnel to enhance monitoring, surveillance and educational services of a generic medical contact center.

Works using MAS are being increasingly conducted to discover more application fields to offer intelligent methods for solving complex problems in a distributed architecture. This paper builds on these works to propose a MAS architecture offering a solution for a complex and large-sized OTLP. The next section is devoted to the mathematical modeling of the SOTLP and to the description of the applied MAS architecture.

\section{SOTLP}

\subsection{Problem Description}

Given a set of facilities, the dimensions and orientation of each facility, and the available area, the SOTLP seeks to determine the optimal placement of the set of facilities within the available space, subject to a set of constraints while optimizing an objective function. In this work, a bi-objective function is considered, which includes quantitative and qualitative measures related to the characteristics of the SOTLP under consideration.

To address the OTLP, the safety and hygiene considerations necessitate a layout organization with a multi-section structure to prevent contamination, to avoid accidents, and to facilitate the movement of material and different actors of the OT. The multi-section structure consists of separated zones (i.e. surgery zone, restricted zone, public zone, etc.), each one having all necessary facilities to operate.

The new variant is formulated with the objective of determining the locations of the sections in the final layout, and the location of facilities and corridors within each section that minimizes the total traveling costs and maximizes the total adjacency rating.

To formulate the mathematical model of the multi-section SOTLP, several assumptions have been considered:

- The facilities have rectangular shapes with unequal areas of known dimensions;

- The movement between facilities is modeled as a rectilinear centroid-to-centroid movement;

- The number of trips, the difficulty of movements, the desirable relationship values between facilities and the cost factors assigned to each entity types are given;

- $\quad$ The orientation of the OT layout is a given parameter; 
- The maximum number of corridors and their orientations are given. In the solution, corridors are used to travel between two facilities in the same section, and to transit from one section to another;

- The location of corridors is a decision variable;

- The corridors are aligned according to the layout orientation to ensure the entrance to the OT unit, the movements between sections, and the exit from the OT unit;

- $\quad$ No relationships of facilities with the outside world are considered;

- A single facility cannot be split among multiple sections.

The main two objectives we aim to optimize in this work are to minimize the total traveling cost (quantitative) and to maximize the total adjacency rating (qualitative). We express these two objectives in the following objective function:

$$
\operatorname{Min}\left[\rho_{1} \sum_{i=1}^{N} \sum_{j=1}^{N} \sum_{k=1}^{K} F_{i j k} D_{i j}\left(\varphi_{i j k} * \sigma_{k}\right)-\rho_{2} \sum_{i=1}^{N} \sum_{j=1}^{N} R_{i j} \mu_{i j}\right],
$$

where $N$ is the set of facilities, $K$ is the set of actors in the OT, $F_{i j k}$ is the traveling frequency from facility $i$ to $j$ by actor $k$ (parameter), $D_{i j}$ is the distance between facility $i$ and $j$ (decision variable), $\varphi_{i j k}$ is the difficulty of the trip according to the provided effort and the requested resources (parameter), $\sigma_{k}$ is the traveling cost corresponding to the actor $k$ (parameter), $R_{i j}$ is the desirability relationship value between facility $i$ and $j$ according to the AEIOUX rating system [21] (parameter), and $\mu_{i j}$ is the proximity coefficient according to the distance between facility $i$ and $j$ (decision variable).

The AEIOUX rating system represents the need for proximities between two facilities, where a strong positive relationship indicates a proximity rank of $A$. The AEIOUX rates are associated to values equal to $A=16$, $E=8, I=4, O=2, U=0$, and $X=-2$, respectively, and are defined according to the international standards in terms of hygiene or decision makers' preferences.

The parameters, decision variables, constraints and results of this mathematical formulation are reported in detail in Ref. [3]. This paper is devoted to the MAS architecture and the new additions or alterations to the principal model.

\subsection{MAS Approach}

In our approach, we used cognitive and communicative agents. Thus, to communicate, agents need a developed language to be able to exchange messages. However, a simple model of message is not enough to tolerate conversations between agents and to allow language acts to fulfill their meaning. For this, we introduced the notion of protocol to support such conversations. The negotiation strategy is proposed based on the Contract Net protocol implemented using the JADE platform that aims to simplify the development of MAS while providing a complete set of services and agents complying with foundation for Intelligent Physical Agents specifications [7].

In this paper, the OT is divided into several departments according to their specialty, (e.g. orthopedics, gynecology, etc.), while each one contains all necessary facilities to be autonomous (e.g. operating rooms, induction room, post-anesthesia care unit, etc.).

The newly adopted approach is modeled in three levels of abstraction (see Figure 1). The first one consists of defining the dimensions and orientation of the plan layout or the initial construction site in which all departments and facilities will be implanted. In the second level, the MILP formulation is used to solve the layout problem for departments first, and to calculate the placement of each one of them in the initial plan layout. Finally, the third level considers the resolution of the MILP for each department in order to determine the optimal locations and orientation for the facilities within.

This three-step decision-making approach can best be handled through an effective and efficient cooperation among multiple agents. To deal with this, we adopt a master/slave architecture where the master 


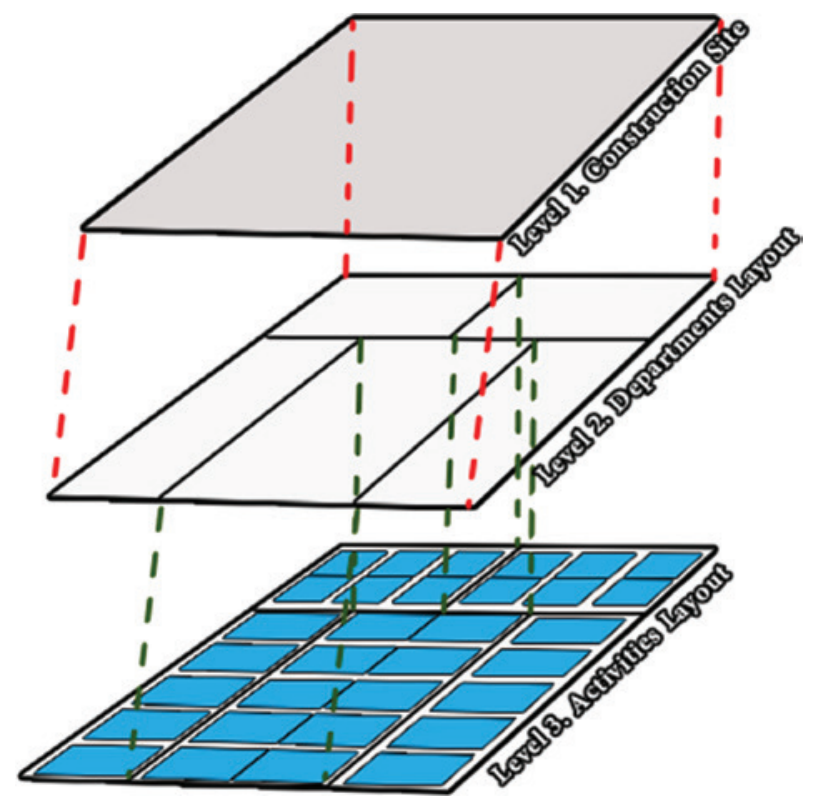

Figure 1: Three-Level FLP Approach.

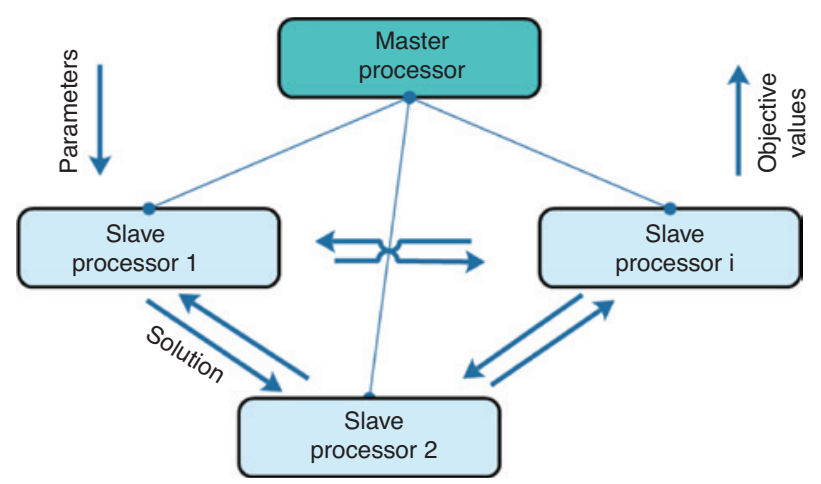

Figure 2: Master/Slave Architecture.

processor (MP) is the coordinator of the optimization process, while the slave processor (SP) is the worker executing tasks coming from the MP. The main task of an MP is to dispatch parameters and orders to each generated SP, which, in parallel, solves the sub-problems and sends back the results with objective value (see Figure 2).

The used algorithm in Figure 1 can be described as follows:

\section{Step 1. Initialization}

JADE platform: The initiator launches the mainContainer, sets up the graphic interface (GUI), initializes program parameters, and registers the MP.

Problem data: Initialize the OT dimension and orientation, fix the number of departments and their dimensions, and determine the list of facilities and their dimensions for each department.

Step 2. Solving the department layout

Once registered, the MP starts solving the OTLP for the list of departments (D) using the objective function (10), and returns the location and the orientation of each department in the plan. These results will be parameters of the next step.

Step 3. Solving each department layout

1. The MP creates and registers an SP for each department and uses the previous results to impose bounding constraint in solving sub-problems. 
2. Each SP first receives a department data (e.g. list of facilities, dimensions, traveling flows, proximity coefficients, etc.) from the MP and starts solving the MILP formulation using the objective function in Eq. (1) for the facilities and corridors in the department.

3. When solved, each SP communicates its optimal layout with the facilities' location and orientation to the other SPs in order to compare their solutions.

4. The SPs send the retained solutions to the MP.

5. The step stops when the MP collects all solutions.

Step 4. Drawing a solution

The result of the collaboration of SPs is an optimal organization of departments in the OT layout and an optimal organization of facilities within each department. The MP sends this solution to the GUI, which draws it and shows it to the decision makers in order to validate it.

Step 5. Agents' destruction

Finally, the MP informs the mainContainer about the end of process and destructs all working agents in the JADE platform.

This study on MAS allowed us to define how the capacity of cognitive agents can be used in a decisionmaking system, namely their intelligence, cooperation and learning.

In addition to the MILP formulation used in Ref. [3] to solve the layout problem for each department, we need to add some parameters, variables and constraints related to the department in order to define their optimal location and orientation in the OT layout. To make our MILP suitable for the three-level approach, these constraints are considered in order to calculate the total interdepartmental traveled distance:

$$
\begin{aligned}
& \operatorname{OrD}_{d}\left(X p_{i j}\right)+\left(1-\operatorname{OrD}_{d}\right)\left(Y p_{i j}\right) \\
& \geq \operatorname{OrD}_{d}\left[\left(x_{i}-x_{c}\right)+\left(x_{c}-x_{j}\right)\right]+\left(1-\operatorname{OrD} D_{d}\right)\left[\left(y_{i}-y_{c}\right)+\left(y_{c}-y_{j}\right)\right] \\
& \forall i \in \mathcal{N}, \forall j \in \mathcal{N}, \forall d \epsilon D, \forall c \in \mathcal{C} \text {, } \\
& \operatorname{OrD}_{d}\left(X p_{i j}\right)+\left(1-O r D_{d}\right)\left(Y p_{i j}\right) \\
& \geq \operatorname{OrD}_{d}\left[\left(x_{i}-x_{c}\right)+\left(x_{j}-x_{c}\right)\right]+\left(1-\operatorname{OrD} D_{d}\right)\left[\left(y_{i}-y_{c}\right)+\left(y_{j}-y_{c}\right)\right] \\
& \forall i \in \mathcal{N}, \forall j \in \mathcal{N}, \forall d \in D, \forall c \in \mathcal{C} \text {, } \\
& \operatorname{OrD}_{d}\left(X p_{i j}\right)+\left(1-\operatorname{OrD}_{d}\right)\left(Y p_{i j}\right) \\
& \geq \operatorname{OrD} D_{d}\left[\left(x_{c}-x_{i}\right)+\left(x_{c}-x_{j}\right)\right]+\left(1-\operatorname{OrD} D_{d}\right)\left[\left(y_{c}-y_{i}\right)+\left(y_{c}-y_{j}\right)\right] \\
& \forall i \in \mathcal{N}, \forall j \in \mathcal{N}, \forall d \epsilon D, \forall c \in \mathcal{C}, \\
& \operatorname{OrD}_{d}\left(X p_{i j}\right)+\left(1-\operatorname{OrD}_{d}\right)\left(Y p_{i j}\right) \\
& \geq \operatorname{OrD}_{d}\left[\left(x_{c}-x_{i}\right)+\left(x_{j}-x_{c}\right)\right]+\left(1-\operatorname{OrD}_{d}\right)\left[\left(y_{c}-y_{i}\right)+\left(x_{j}-x_{c}\right)\right] \\
& \forall i \in \mathcal{N}, \forall j \in \mathcal{N}, \forall d \epsilon D, \forall c \in \mathcal{C} \text {, } \\
& \operatorname{OrD}_{d}\left(Y p_{i j}\right)+\left(1-\operatorname{OrD}_{d}\right)\left(X p_{i j}\right) \\
& \geq \operatorname{OrD}_{d}\left(y_{i}-y_{j}\right)+\left(1-\operatorname{OrD} D_{d}\right)\left(x_{i}-x_{j}\right) \\
& \forall i \in \mathcal{N}, \forall j \in \mathcal{N}, \forall d \in D \text {, } \\
& \operatorname{OrD}_{d}\left(Y p_{i j}\right)+\left(1-\operatorname{OrD}_{d}\right)\left(X p_{i j}\right) \\
& \geq \operatorname{OrD}_{d}\left(y_{j}-y_{i}\right)+\left(1-\operatorname{OrD}_{d}\right)\left(x_{j}-x_{i}\right) \\
& \forall i \in \mathcal{N}, \forall j \in \mathcal{N}, \forall d \epsilon D \text {, }
\end{aligned}
$$

where $X p_{i j}$ and $Y p_{i j}$ are variables representing, respectively, the horizontal and vertical distances between two departments $i$ and $j ; x_{i} / x_{j}$ and $y_{i} / y_{j}$ are the used variables to determine the location of a department; $x_{c} / y_{c}$ are the variables used to detrmine the location of a corridor; and $\operatorname{Or} D_{d}$ refers to each department orientation, 
which takes 1 if the length of department $d$ is parallel to the $y$-axis (vertical orientation) and 0 otherwise. We can obtain it by using the following equations:

$$
\begin{gathered}
W_{d}=\vartheta_{d} \operatorname{Or} D_{d}+\theta_{d}\left(1-O r D_{d}\right) \quad \forall d \epsilon D, \\
L_{d}=\vartheta_{d}+\theta_{d}-D_{d} \quad \forall d \epsilon D,
\end{gathered}
$$

where

- $\quad \vartheta_{d}$ : length of department $d$ (parameter);

- $\theta_{d}$ : width of department $d$ (parameter);

- $\quad L_{d}: X$-length of department $d$ depending on whether $\vartheta_{d}$ or $\theta_{d}$ is parallel on the $x$-axis (decision variable);

- $\quad W_{d}: Y$-length of department $d$ depending on whether $\vartheta_{d}$ or $\theta_{d}$ is parallel on the $y$-axis (decision variable).

The objective function in the second level is formulated in Eq. (10), where $F_{l h k}, D_{l h}, \varphi_{l h k}, R_{l h}$ and $\mu_{l h}$ are, respectively, the inter-departments' traveling frequency (see Appendix A), traveling distance, traveling difficulty (see Appendix A), relationship, and adjacency coefficient (see Appendix A):

$$
\operatorname{Min} F D=\rho_{1} \sum_{l=1}^{D} \sum_{h=1}^{D} \sum_{k=1}^{4} F_{l h k} D_{l h}\left(\varphi_{l h k} * \sigma_{k}\right)-\rho_{2} \sum_{l=1}^{D} \sum_{h=1}^{D} R_{l h} \mu_{l h} .
$$

\section{AOTLP}

\subsection{Problem Description}

To the best of our knowledge, hospital layout planning is still being addressed as SFLP, where material handling flows stay invariant over a long time. As far as we know, hospitals and specifically OT are volatile. Under such conditions, some parameters such as patients demand are not stable and the facilities must be adaptive to demand change requirements. For these reasons, a static layout analysis would not be sufficient.

To overcome the disadvantages of the SFLP, we provide an adaptive formulation for OTLP (AOTLP), with the objective of minimizing the interdepartmental travel costs among facilities, maximizing the adjacency rating, and minimizing the rearrangement costs [Eq. (11)]. The AOTLP is solved by studying an individual layout for each distinctive period based on patient demands, subject to a set of distance constraints, available areas, and non-overlapping facilities according to international medical standards and specifications.

In order to model the OTLP in an uncertain environment, two different solutions can be proposed. The first approach consists of finding in one single decision a robust OT layout that is the optimal layout for all periods and that could not be rearranged in later periods. The second one is an adaptive solution, which consists of generating a layout plan for each period of the planning horizon. Here, the layout will be rearranged in a way that it satisfies the patient demands of each period. Table 1 summarizes the differences between the robust approach of Chraibi et al. [4] and our adaptive approach.

Table 1: Robust Layout vs. Adaptive Layout.

\begin{tabular}{lll}
\hline & Robust layout & Adaptive layout \\
\hline Facilities & Fixed facilities only & Movable and non-movable facilities \\
Layout & Best possible layout for entire planning horizon & Adaptations for each period in order to satisfy demand \\
Costs & Building fixed facilities, travelling costs & $\begin{array}{l}\text { Building fixed and movable facilities, facilities rearrangement to } \\
\text { adapt layout, travelling costs }\end{array}$ \\
Objective & Minimize travelling costs, maximize adjacency & $\begin{array}{l}\text { Minimize travelling costs, maximize adjacency, minimize layout } \\
\text { rearrangement costs }\end{array}$ \\
& &
\end{tabular}


Traditional FLP approaches consider a planning horizon generally divided into periods of weeks, months or years. Choosing the period length has an impact on the final results. In fact, in a short period, the flows are fairly constant and there will not have a need for layout rearrangement and the use of adaptive layout analysis may not be justified. In contrast, a long period will lead to prohibitive rearrangement costs and this goes against the objective of cost minimization. This choice should be made according to the studied system based on demand and the delivered asset and service.

In the case of our MAS approach, this paper will focus on the AOTLP and present just the adopted architecture and novelties compared to the developed MILP for the problem in Ref. [4]. The developed MILP aims to minimize the traveling costs for the considered entities (doctors, patients, and medical and non-medical staff) for each period in the planning horizon, to maximize the adjacency rating between dependent facilities and to minimize rearrangement costs generated by moving appropriated equipment for each room specialty [Eq. (11)]. This model provides an optimal solution for problems with $<17$ facilities, which represents the size of one simple department in large hospitals.

$$
\operatorname{Min}\left[\sum_{t=1}^{P} \sum_{i=1}^{N} \sum_{j=1}^{N} \sum_{k=1}^{K} F_{i j k t} D_{i j t}\left(\varphi_{i j k} * \sigma_{k}\right)-\sum_{t=1}^{P} \sum_{i=1}^{N} \sum_{j=1}^{N} R_{i j} \mu_{i j t}+\sum_{t=1}^{P} \sum_{i=1}^{N} \sum_{j=1}^{N} A_{i j} C_{i j} r_{i j t}\right],
$$

where $A_{i j}$ is a binary parameter equals to 1 if facilities $i$ and $j$ can be exchanged, and 0 otherwise; $C_{i j}$ is the relocation cost of facility $i$ if exchanged with $j$ (parameter); and $r_{i j t}$ is a binary variable equals to 1 if facility $i$ is exchanged with $j$ in period $t$, and 0 otherwise.

\subsection{MAS Approach}

To solve the AOTLP, we introduce a new three-level approach, which is illustrated in Figure 3. The first step defines the dimensions and orientation of the plan layout or the initial construction site in which all departments and facilities will be implanted and divides it into equal-sized departments corresponding to number

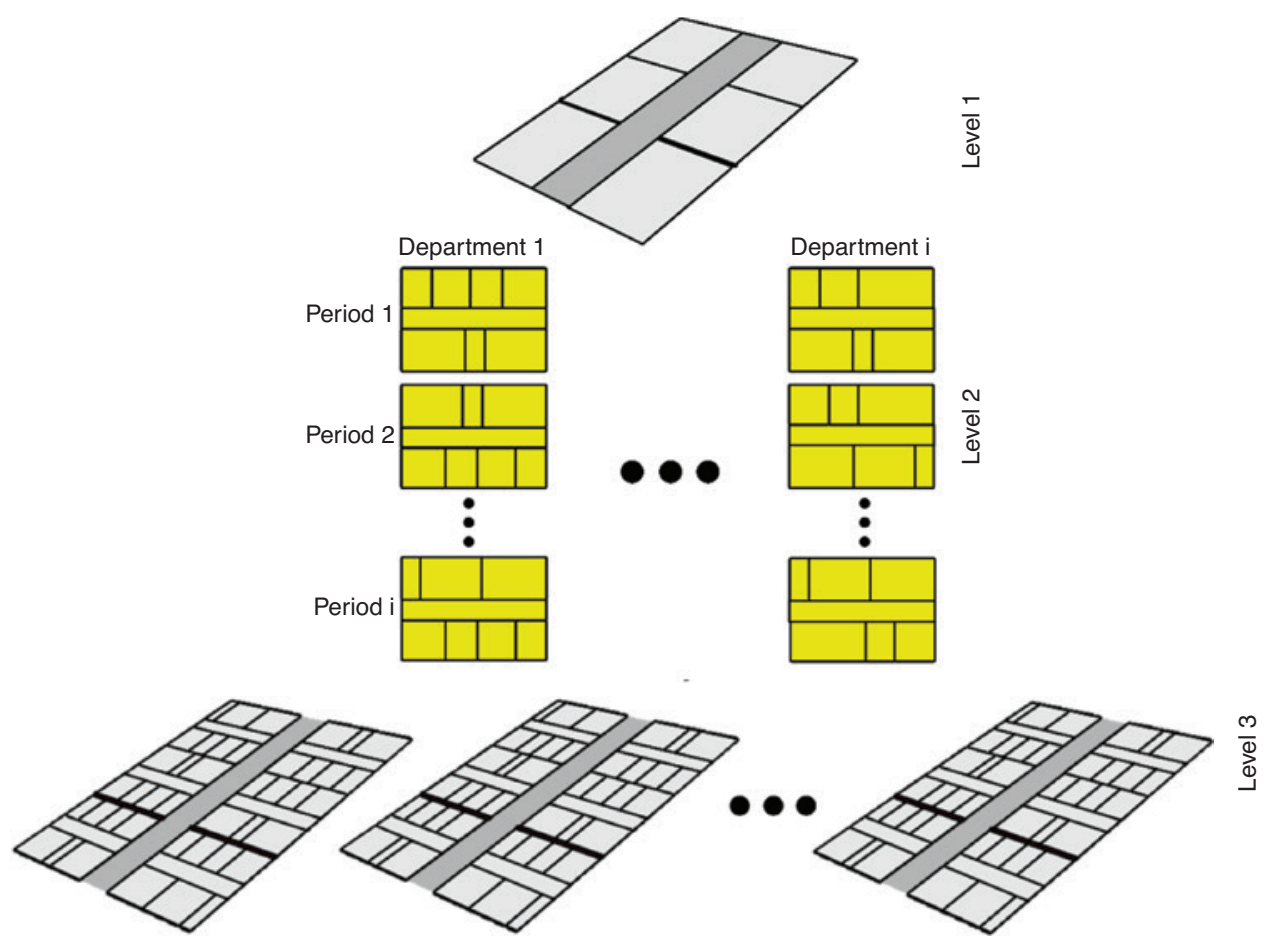

Figure 3: Three-Level Adaptive FLP Approach. 
of OT specialties. For each department, the second level consists of solving the adaptive layout problem for all periods of the planning horizon using the objective function in Eq. (11) and the MILP in Ref. [4] in order to determine the optimal location and orientation for each facility within the department. Considering these solutions, the optimal OT layout in a period $t$ is addressed by using all resulting departments in period $t$. For this, departments are assigned to the defined locations - in the first step - according to the total relations rate (TRR) calculated in Eq. (12). To assign departments, the quadratic assignment problem (QAP) formulation in Eqs. (13)-(15) is adopted to determine the location of each department in the final layout while minimizing the traveling costs and maximizing the adjacency of the departments.

$$
\mathrm{TRR}_{h k}=\sum_{i=1}^{N} \sum_{j=1}^{N}\left(r_{i j h k}+r_{j i h k}\right) \quad \forall h \epsilon D, \forall k \epsilon D,
$$

where

- $\quad D$ is the set of departments.

- $\quad r_{i j h k}$ is the relationship value that expresses the need for proximity between the facility $i$ in the department $h$ and the facility $j$ in the department $k$ by using the AEIOUX rating system.

$$
\operatorname{Min} \sum_{h=1}^{D} \sum_{k=1}^{D} \sum_{m=1}^{D} \sum_{l=1}^{D} C_{h k} D_{h k} X_{h l} X_{k m}-\sum_{h=1}^{D} \sum_{k=1}^{D} T R R_{h k} \mu_{h k} .
$$

Subject to

$$
\begin{gathered}
\sum_{h=1}^{D} X_{h l}=1 \quad l=1, \ldots, D ; \\
\sum_{k=1}^{D} X_{k m}=1 \quad m=1, \ldots, D ;
\end{gathered}
$$

- $\quad X_{h l}$ : a binary variable equal to 1 if department $h$ is assigned to location $l$, and 0 otherwise;

- $\quad \mu_{h k}$ : the adjacency coefficient that expresses the desirability of locating adjacent departments next to each other. It will be 1 if two departments are fully adjacent, 0.5 if they belong to the same section, or 0 if they are located in different sections;

- $C_{h k}$ : the traveling cost between department $h$ and $k$;

- $\quad D_{h k}$ : the distance between department $h$ and $k$.

Constraints in Eqs. (14) and (15) are used to ensure that each department is assigned to only one location and that each location contains only one department.

This three-step decision-making approach can be best handled through an effective and efficient cooperation of multiple agents in the second level to find the best layout, and a negotiation protocol in the third level to allow each agent to occupy the best location in the OT layout. For this, we adopt a master/slave/subslave architecture, where the MP is the coordinator of the optimization process, the SP is a worker executing tasks coming from MP and at the same time a coordinator of sub-SPs (Sub_SPs) attached to him, while the Sub_SP is a worker executing tasks coming from the SP (see Figure 4).

The main task of an MP is first to generate as many SPs as departments (i.e. specialties) and then to instruct each SP to solve a department layout problem for all the planning horizon periods. The main task of each SP is to generate as many Sub_SPs as periods and then to instruct each Sub_SP to solve the FLP for each period of the planning horizon. At the bottom of the hierarchy, Sub_SPs receive all required data to solve the layout problem and start the optimization process. Each group of Sub_SPs (i.e. attached to the same SP) compare their solutions in order to count the number of facility rearrangements (i.e. facilities having exchanged their locations from a period to another) in the whole planning horizon to be calculated in the 


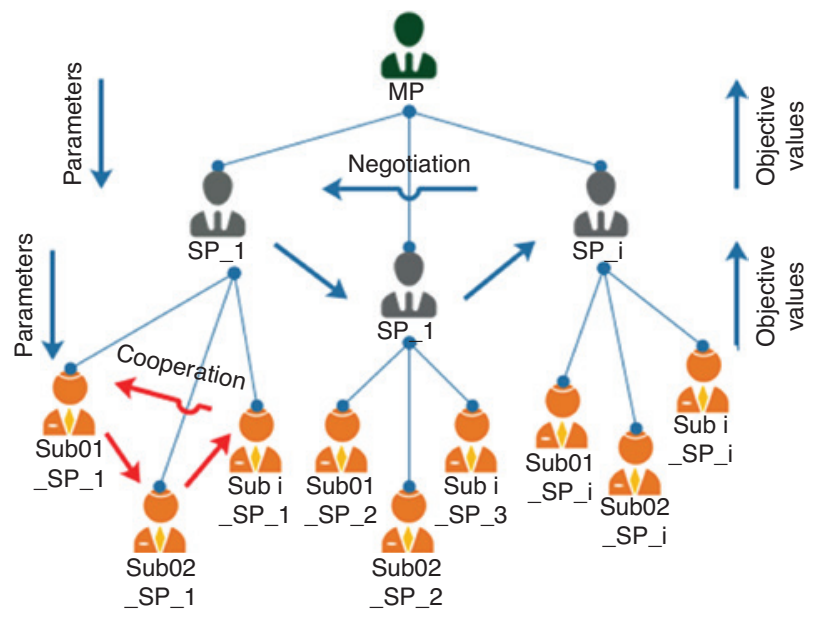

Figure 4: Master/Slave/Sub-slave Architecture.

objective function in Eq. (11). When all departments' layouts are solved, the SPs start a negotiation phase to assign the departments to the available locations in the final OT layout for each period. Finally, the MP addresses all the obtained layouts (i.e. a layout per period) and presents the solutions to decision makers.

The developed interaction between agents to solve AOTLP can be described in the following algorithm:

\section{Step 1. Initialization}

JADE platform: The initiator launches the mainContainer, sets up the GUI, initializes program parameters, and registers the MP.

Problem data: Initialize the OT dimension and orientation, fix the number of departments and their dimensions, and determine the list of facilities and their dimensions for each department.

Step 2. Dividing the initial OT plan

[1] The MP divides the OT into equal-sized departments according to the number of specialties and as imposed by hospital managers.

[2] The MP creates and registers an SP for each department.

[3] The MP sends the required data for each department (e.g. list of facilities, dimensions, traveling flows, proximity coefficients, etc.) to an SP.

[4] The MP is on standby mode waiting for results coming from SPs.

Step 3. Each SP solves a department layout

[1] For each period in the planning horizon, the SP generates a Sub_SP to solve the department layout problem for this period.

[2] Each Sub_SP starts solving the MILP formulation using the function in Eq. (11) for the facilities and corridors in the department.

[3] The Sub_SPs start the cooperation phase by comparing their results in order to determine the total rearrangement cost.

[4] The Sub_SPs send the optimal layouts with facilities' location and orientation to the SP.

Step 4. Solving the whole OT layout

[1] The SPs receive answer from their Sub_SPs.

[2] The SPs start the negotiation phase to determine the best assignment for each department using the QAP formulation [Eq. (13)] according to their calculated TRR.

[3] The SPs send the final solution to the MP.

[4] The step stops when the MP collects all solutions.

Step 5. Presenting the final solution

The result of the cooperation of Sub_SPs and negotiation of SPs is a set of optimal OT layouts for the whole planning horizon.

[1] The MP then sends these solutions to the GUI that draws it and shows it to the decision makers to validate it.

Step 6. Agents' destruction

[1] Finally, the MP informs the mainContainer about the end of process.

[2] The MP destructs all working agents in the JADE platform. 
In this formulation, we used two agent communication protocols. The first is cooperation, where agents try to find a solution that minimizes the total rearrangement cost in a way that it satisfies the all periods' surgery demand. The second protocol is negotiation, in which each agent competes to find a location that satisfies its own benefit. This allows us to exploit agent properties to find efficient solutions in a reasonable time.

\section{Experiments and Results}

In this section, we present the experimentations with the proposed MA algorithm based on MILP formulation using a set of previously generated data. We exploit the small instances used for the exact formulation in Refs. [3, 4] for the SOTLP and AOTLP to constitute the needed departments, each of no more than 17 facilities to make possible the use of the developed MILP by the MAS.

For running the MILP, we used (IBM, Armonk, NY, USA) ILOG CPLEX Optimization Studio 12.5 software to solve the model using Windows 7 platform, Intel 5 Core i5-3380M CPU@ 2.90 GHz and 8 Gb of RAM, while the MAS platform has been implemented using JAVA language and modeled using UML. An example of used data can be found in Appendix A.

First, the program structure has been modeled using the package diagram provided by UML (Figure 5). We divide it into three packages: the first contains all the required class to model the JADE agents, the second models the different OT components (OT, departments, facilities and layout solution), while the third package regroups the needed classes to design the GUI.

\subsection{SOTLP}

Experiments have been conducted on the SOTLP by configuring OT instances with a combination of departments of generally 16 facilities and 13 facilities. Several OT configurations have been constituted with medium sizes (e.g. 29 and 54 facilities) and large sizes (e.g. 87, 116, 145 and 174 facilities). They all were solved using MAS of one MP and as many departments as SPs. An example of larger-sized OTLP with 12 departments with 174 facilities is solved using 13 agents (i.e. 12 SPs and 1 MP). The number of facilities is no longer a limitation as the sub-tasks are feasible MILP for each SP. We decided to stop our experiments at

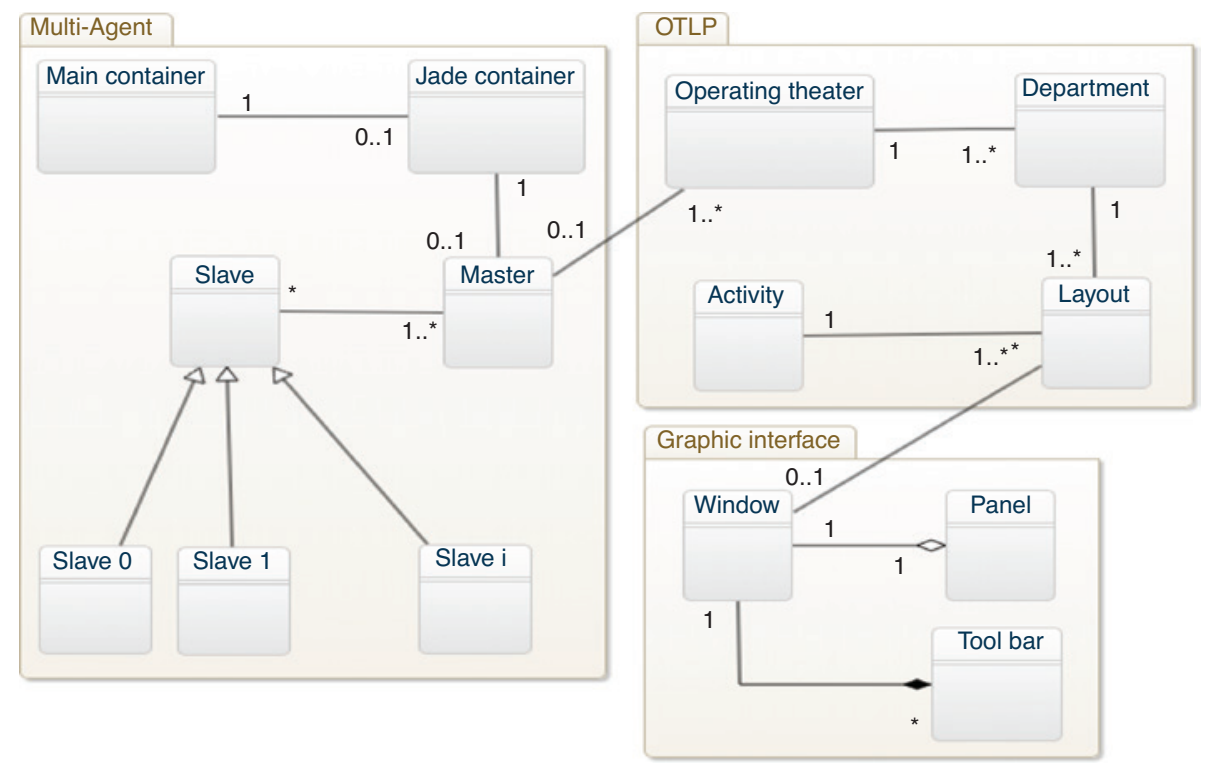

Figure 5: MAS Package Diagram. 
OT with 174 facilities to deal with realistic OT sizes. Table 2 presents a description of the rooms list in each instance size.

Table 3 provides some computation results obtained using the MILP model in Ref. [3]. The limit of this MILP is 18 facilities; beyond this value, optimality is not guaranteed. Compared to these obtained results, our MAS gives an efficient solution for larger-sized problems in a shorter time. For example, a problem with 16 facilities took $623 \mathrm{~s}$ with simple MILP, while MAS solved in the same duration a problem with 16 facilities. This shows the performance of this MA approach in terms of timeliness and capacity.

To be feasible, each agent is constrained to respect the limitation of the MILP model (i.e. 18 facilities); otherwise, this agent will not be able to return a solution. Table 4 provides the results of solving multi-department OTLP using MAS with different instance sizes. The first column gives the number of facilities in the OT, the second represents the number of used SPs to solve this problem size (number of SPs=number of departments), while the third one gives the processing time in different running iterations to verify the ability of agents to learn from their previous experiences. The MAS has been executed several times on the same instance to examine the agents' characteristics and properties in each iteration. We observed that the MAS took less time for solving the problem than in the previous run, while the objective value remained fixed. This improvement on the execution time is due to the agents' learning capacity. Figure 6 shows that this execution time regresses while repeating calculation for each problem size, while Figure 7 presents the final results for an OT problem with 87 facilities assigned to six departments.

Table 2: Description of OT Instances According to OT Size.

\begin{tabular}{ll}
\hline Instance size & Rooms list \\
\hline 11 Facilities & $\begin{array}{l}4 \text { Operating rooms, } 1 \text { scrub room, } 1 \text { PACU, } 1 \text { decontamination, } 1 \text { sterile arsenal, } 1 \text { cleaning room and } \\
2 \text { corridors }\end{array}$ \\
$\begin{array}{l}4 \text { Facilities } \\
\text { 13 Operating rooms, } 2 \text { induction rooms, } 1 \text { scrub room, } 1 \text { PACU, } 1 \text { decontamination, } 1 \text { sterile arsenal, } 1 \text { cleaning } \\
\text { room and } 2 \text { corridors }\end{array}$ \\
& $\begin{array}{l}4 \text { Operating rooms, } 2 \text { induction rooms, } 2 \text { scrub room, } 1 \text { PACU, } 1 \text { decontamination, } 1 \text { sterile arsenal, } 1 \text { cleaning } \\
\text { room, } 1 \text { changing room, } 1 \text { rest room and } 2 \text { corridors }\end{array}$ \\
\hline
\end{tabular}

Table 3: Obtained Results in Ref. [3].

\begin{tabular}{lrr}
\hline Instance size & Objective value & Time (s) \\
\hline 11 Facilities & 124,523 & 7 \\
13 Facilities & 233,672 & 25 \\
16 Facilities & $3,263,600$ & 623 \\
17 Facilities & $7,535,150$ & 4173 \\
18 Facilities & $7,635,890$ & 19,851 \\
\hline
\end{tabular}

Table 4: Processing Time for Different Instance Sizes of the Static Formulation.

\begin{tabular}{|c|c|c|c|c|}
\hline \multirow{2}{*}{$\begin{array}{l}\text { Number of } \\
\text { facilities }\end{array}$} & \multirow{2}{*}{$\begin{array}{l}\text { Number } \\
\text { of agents }\end{array}$} & \multicolumn{3}{|c|}{ Time (s) } \\
\hline & & $1^{\text {st }}$ run & $2^{\text {nd }}$ run & $3^{\text {rd }}$ run \\
\hline 29 & 2 & 194 & 188 & 163 \\
\hline 58 & 4 & 389 & 372 & 332 \\
\hline 87 & 6 & 517 & 506 & 489 \\
\hline 116 & 8 & 672 & 669 & 657 \\
\hline 145 & 10 & 927 & 908 & 888 \\
\hline 174 & 12 & 1276 & 1137 & 1086 \\
\hline
\end{tabular}




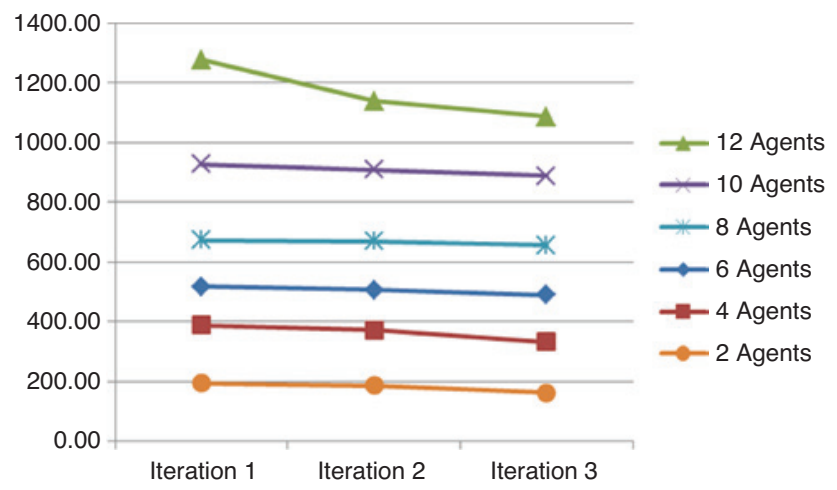

Figure 6: Processing Time Regression.

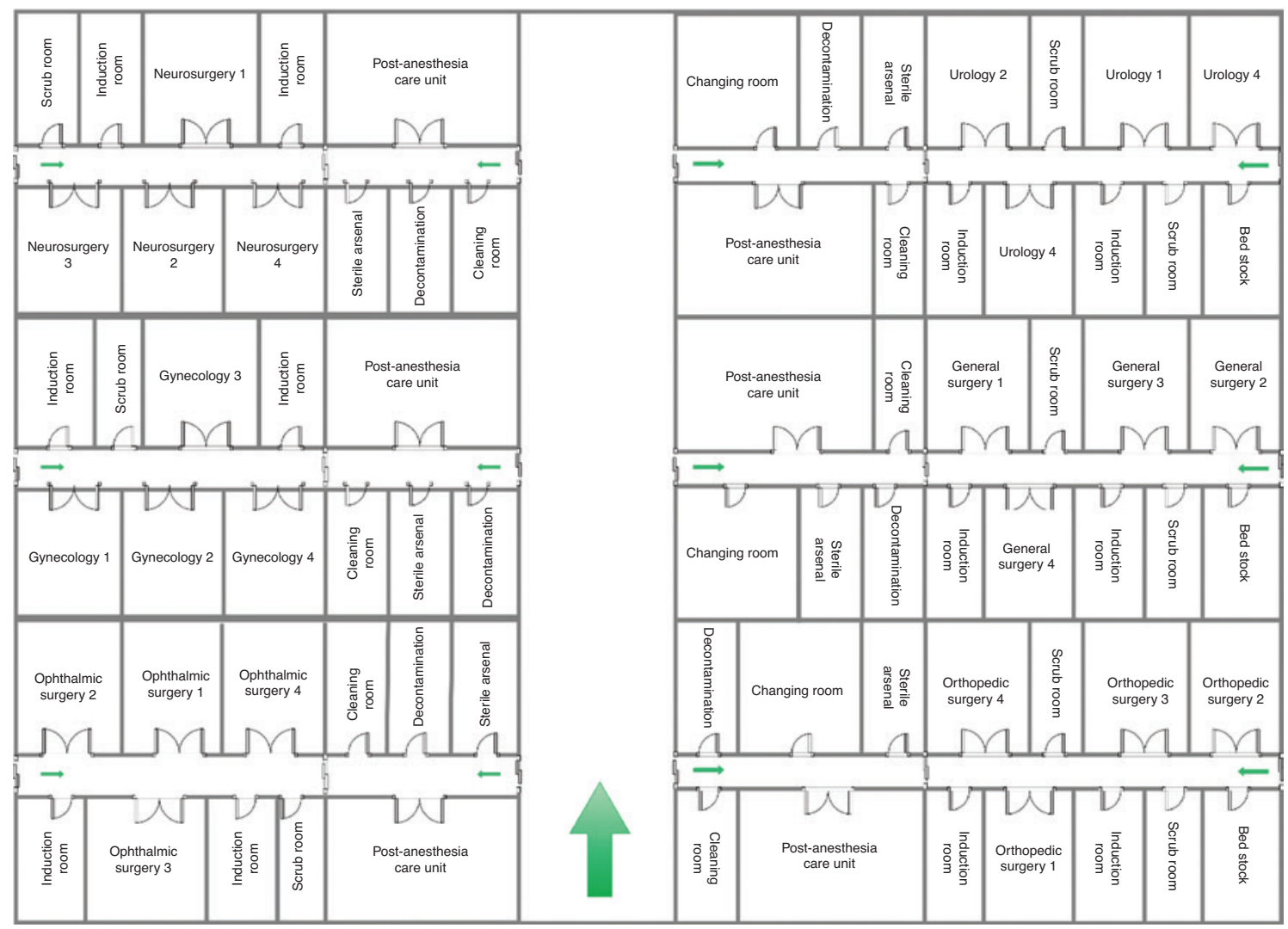

Figure 7: SOTLP: Final OT Layout.

\subsection{AOTLP}

Experiments have been conducted on the AOTLP by configuring OT instances with a combination of departments of generally 13 facilities and 11 facilities. Several OT configurations have been constituted with medium sizes (e.g. 52 facilities) and large sizes (e.g. 78 and 104 facilities). They were all solved using MAS of one MP, as many departments as SPs, and as many periods as Sub_SPs under each SP (e.g. the final number of agents for an OT of four departments in a planning horizon of three periods is $1+4^{\star} 3=13$ ).

The adaptive approach consists of exchanging rooms' or departments' specialty from a period to another while minimizing rearrangement costs in a way that two departments can exchange their specialty and move 
the needed equipment and materials from one to the other to satisfy patient demand. Assumptions used in this formulation were approved by a senior manager of the OT of the hospital in Roanne city (in France).

The proposed MILP model solved small instances with 17 facilities as maximum value with a planning horizon of three and six periods. Beyond these values, an optimal solution will not be guaranteed. Table 5 presents a part of the obtained solutions using just the MILP model.

In this work, we consider a multi-disciplinary OT, which means that each department is affected to a surgical specialty. To rearrange the OT layout, we adopt the exchange of the surgical specialty between departments and the surgery operations' affectation between operating rooms, as restructuration of the whole OT after each period can be very expensive. In this case, the OT will keep the first layout configuration and room specialty could be exchanged at the end of each period to satisfy patient demands.

Compared to the obtained results in Table 5, our MAS gives an efficient solution for larger-sized problems in a shorter time. For example, a problem with 17 facilities took $3935.89 \mathrm{~s}$ with a simple MILP, while MAS solved problems with larger sizes in less than this duration. This shows the performance of this MAS approach in terms of timeliness and capacity. In addition to this, the MILP capacity is limited to a planning horizon of six periods, while in MAS we investigate problems with 9 and 12 periods in a reasonable execution time.

To be feasible, each agent is constrained to respect the limitation of the MILP model (i.e. 17 facilities); otherwise, this agent will not be able to return a solution. Table 6 provides the results of solving AOTLP using MAS with different instance sizes and different planning horizon repartitions. The first and second columns give the number of departments and the number of facilities in the OT, whereas the third represents the number of used agents to solve this problem size according to the number of periods in the fourth column.

Table 5: Obtained Results for AOTLP Using MILP Model.

\begin{tabular}{lrrr}
\hline Instance size & Number of periods & Objective value & Time (s) \\
\hline 11 Facilities & 3 & 288,650 & 1.22 \\
& 6 & 296,700 & 5.35 \\
13 Facilities & 3 & 333,050 & 26.04 \\
& 6 & 376,300 & 32.46 \\
16 Facilities & 3 & 403,430 & 784.44 \\
17 Facilities & 6 & 478,300 & 1055.57 \\
& 3 & 912,350 & 3935.89 \\
& 6 & 987,550 & 5197.13 \\
\hline
\end{tabular}

Table 6: Processing Time for Different Instance Sizes of the Adaptive Formulation.

\begin{tabular}{|c|c|c|c|c|c|c|}
\hline \multirow{2}{*}{$\begin{array}{l}\text { Number of } \\
\text { departments }\end{array}$} & \multirow{2}{*}{$\begin{array}{r}\text { Number of } \\
\text { facilities }\end{array}$} & \multirow{2}{*}{$\begin{array}{r}\text { Number } \\
\text { of agents }\end{array}$} & \multirow{2}{*}{$\begin{array}{r}\text { Number } \\
\text { of periods }\end{array}$} & \multicolumn{3}{|c|}{ Time (s) } \\
\hline & & & & $1^{\text {st }}$ run & $2^{\text {nd }}$ run & $3^{\text {rd }}$ run \\
\hline \multirow[t]{4}{*}{4} & 52 & 13 & 3 & 769 & 654 & 607 \\
\hline & & 25 & 6 & 1059 & 947 & 858 \\
\hline & & 37 & 9 & 1472 & 1441 & 1320 \\
\hline & & 49 & 12 & 2554 & 2501 & 2386 \\
\hline \multirow[t]{4}{*}{6} & 78 & 19 & 3 & 1083 & 971 & 806 \\
\hline & & 37 & 6 & 1676 & 1586 & 1473 \\
\hline & & 55 & 9 & 2752 & 2690 & 2519 \\
\hline & & 73 & 12 & 3342 & 3216 & 3104 \\
\hline \multirow[t]{4}{*}{8} & 104 & 25 & 3 & 1244 & 1194 & 1031 \\
\hline & & 49 & 6 & 2973 & 2846 & 2782 \\
\hline & & 73 & 9 & 4016 & 3933 & 3813 \\
\hline & & 97 & 12 & 5329 & 5202 & 5182 \\
\hline
\end{tabular}



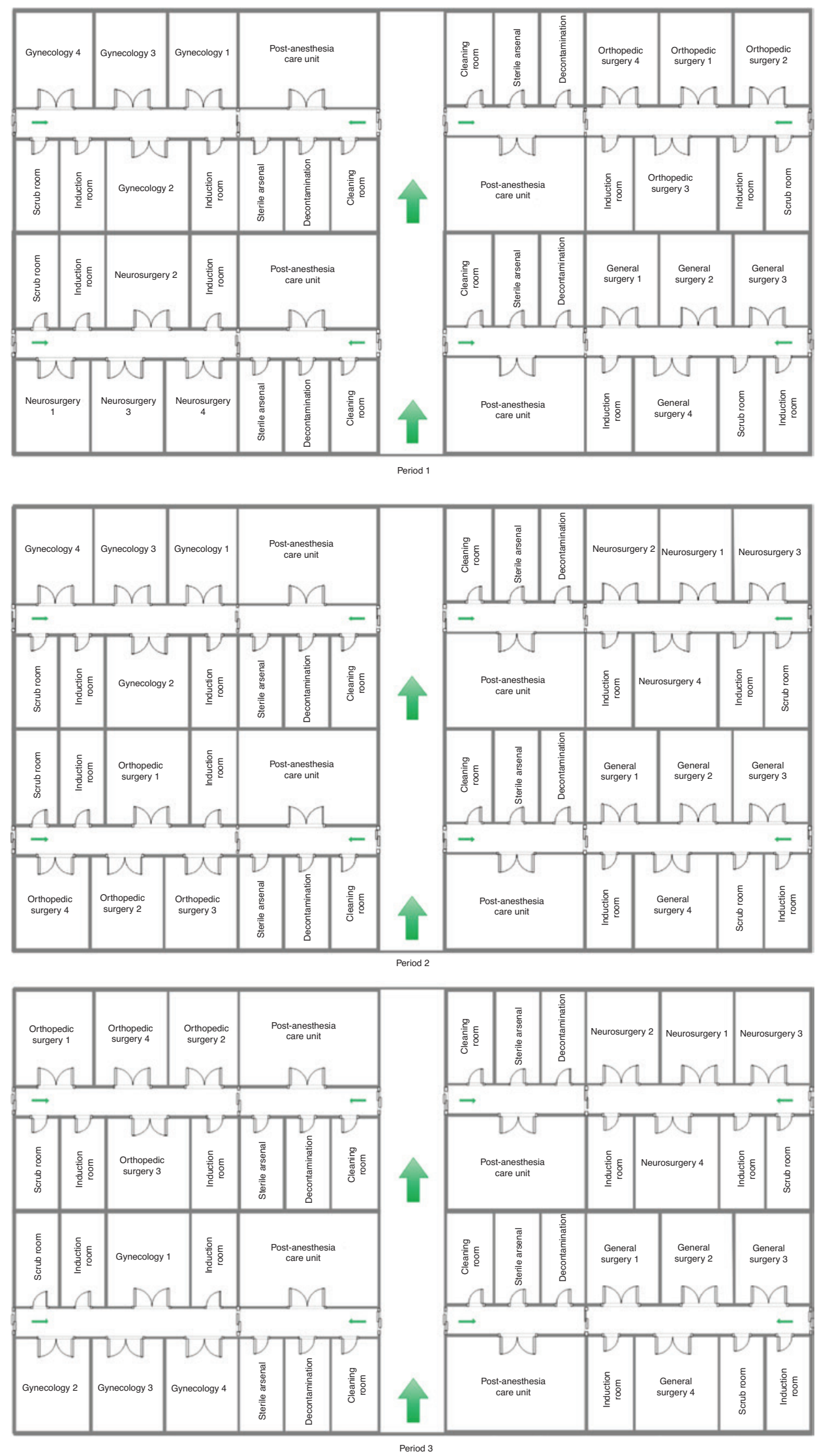

Figure 8: AOTLP: Final OT Layout. 
The last column presents the processing time in different running iterations. We observed running time to also regress in each execution due to the agents' learning capacity. As in the SOTLP, the number of facilities we tested here is not a limitation. We can make the size $>104$ by increasing the number of agents. Figure 8 presents the results for an OT problem with 52 facilities assigned to four departments in a planning horizon of three periods. From the three obtained layouts, we can observe the reallocation of facilities within the OT at different periods to ensure optimality and adequacy for various surgical demands. These assumptions and results were viewed and approved by a senior manager of the OT of the hospital in Roanne city (in France).

\section{Conclusion}

This paper presents a novel approach using a MAS where agents' skills are exploited to cover a wide research space, to accelerate the decision-making process and to deal with real-life problem sizes. This decisionmaking tool is based on several MILP models for solving the FLP and considers two types of environments with deterministic and variant patient demand. The proposed methods were tested on a large-sized OTLP for which exact methods were unable to give solutions, and which represents a real case of OT in big hospitals.

MAS has a very efficient capacity for solving large-sized OTLP using different communication protocols (i.e. cooperation, collaboration and negotiation). This paper presents some of the obtained results while running our MAS on problems with sizes up to 165 facilities, but not necessarily limited to this size. In terms of processing time, final solutions were obtained in reduced time compared to those obtained with exact methods.

In further works, other OT structures will be considered, namely the multi-floor and multi-line layouts in deterministic and variant environments. MAS will be applied to these problems to make our tool suitable to different hospital configurations. Furthermore, the combination of MAS performance and meta-heuristic features could be an interesting area to explore as a way to perform our approach and to solve more complex problems, namely FLP with non-regular shapes, with input/output points, with fixed obstacles, etc.

\section{Appendix A}

Example of Used Data for the Resolution of OTLP.

Appendix Table 1: Traveling Frequency in a Small OT.

\begin{tabular}{|c|c|c|c|c|c|c|c|c|c|c|}
\hline & Facility & $k$ & 0 & 1 & 2 & 3 & 4 & 5 & 6 & 7 \\
\hline \multirow[t]{4}{*}{0} & Outdoor & 1 & & 0 & 0 & 0 & 105 & 0 & 0 & 0 \\
\hline & & 2 & & 210 & 0 & 0 & 0 & 0 & 0 & 105 \\
\hline & & 3 & & 210 & 0 & 0 & 0 & 0 & 0 & 0 \\
\hline & & 4 & & 210 & 0 & 0 & 0 & 0 & 0 & 0 \\
\hline \multirow[t]{4}{*}{1} & Induction1 & 1 & 0 & & 0 & 0 & 0 & 0 & 0 & 0 \\
\hline & & 2 & 0 & & 120 & 90 & 0 & 0 & 105 & 0 \\
\hline & & 3 & 105 & & 60 & 45 & 0 & 0 & 0 & 0 \\
\hline & & 4 & 0 & 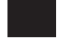 & 60 & 45 & 0 & 0 & 0 & 0 \\
\hline \multirow[t]{4}{*}{2} & OR1 & 1 & 0 & 0 & & 0 & 0 & 0 & 0 & 0 \\
\hline & & 2 & 0 & 0 & & 0 & 0 & 60 & 60 & 0 \\
\hline & & 3 & 0 & 0 & & 0 & 0 & 60 & 0 & 0 \\
\hline & & 4 & 0 & 0 & & 0 & 0 & 60 & 0 & 0 \\
\hline \multirow[t]{4}{*}{3} & OR2 & 1 & 0 & 0 & 0 & & 0 & 0 & 0 & 0 \\
\hline & & 2 & 0 & 0 & 0 & & 0 & 45 & 45 & 0 \\
\hline & & 3 & 0 & 0 & 0 & & 0 & 45 & 0 & 0 \\
\hline & & 4 & 0 & 0 & 0 & & 0 & 45 & 0 & 0 \\
\hline
\end{tabular}


Appendix Table 1 (continued)

\begin{tabular}{|c|c|c|c|c|c|c|c|c|c|c|}
\hline & Facility & $k$ & 0 & 1 & 2 & 3 & 4 & 5 & 6 & 7 \\
\hline \multirow[t]{4}{*}{4} & Scrub room1 & 1 & 0 & 0 & 60 & 45 & & 0 & 0 & 0 \\
\hline & & 2 & 105 & 0 & 0 & 0 & & 0 & 0 & 0 \\
\hline & & 3 & 0 & 0 & 0 & 0 & & 0 & 0 & 0 \\
\hline & & 4 & 0 & 0 & 0 & 0 & 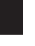 & 0 & 0 & 0 \\
\hline \multirow[t]{4}{*}{5} & PACU & 1 & 0 & 0 & 0 & 0 & 0 & & 0 & 0 \\
\hline & & 2 & 105 & 0 & 0 & 0 & 0 & & 0 & 0 \\
\hline & & 3 & 0 & 0 & 0 & 0 & 0 & & 0 & 0 \\
\hline & & 4 & 105 & 0 & 0 & 0 & 0 & 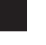 & 0 & 0 \\
\hline \multirow[t]{4}{*}{6} & Decontamination & 1 & 0 & 0 & 0 & 0 & 0 & 0 & & 0 \\
\hline & & 2 & 210 & 0 & 0 & 0 & 0 & 0 & & 210 \\
\hline & & 3 & 0 & 0 & 0 & 0 & 0 & 0 & & 0 \\
\hline & & 4 & 0 & 0 & 0 & 0 & 0 & 0 & 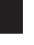 & 0 \\
\hline \multirow[t]{4}{*}{7} & Sterile arsenal & 1 & 0 & 0 & 0 & 0 & 0 & 0 & 0 & \\
\hline & & 2 & 0 & 105 & 60 & 45 & 0 & 0 & 0 & \\
\hline & & 3 & 0 & 0 & 0 & 0 & 0 & 0 & 0 & \\
\hline & & 4 & 0 & 0 & 0 & 0 & 0 & 0 & 0 & \\
\hline
\end{tabular}

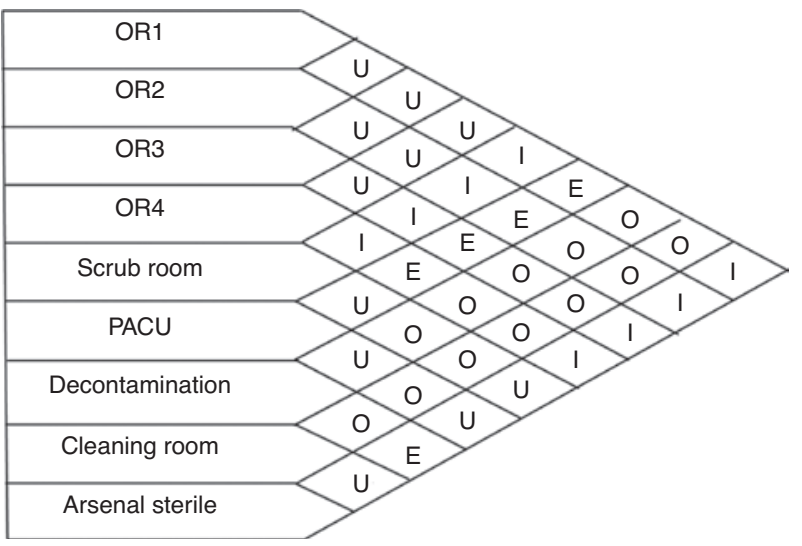

Figure 9: Example of AEIOUX Rating.

Appendix Table 2: Trip Difficulty Rating Based on Movement Description and Entity Types.

\begin{tabular}{llrl}
\hline Type & Entities & $\boldsymbol{\varphi}_{\text {lhk }}$ & Movement description \\
\hline 1 & 1.5 & Movement requires some resources \\
2 & Patients & 1 & Completely independent \\
3 & Doctors & 1.5 & Movement done with a patient or some equipment \\
& Medical staff & 1 & Movement done freely \\
4 & 1.5 & Movement done with a patient or some equipment \\
& Non-medical staff & 1 & Movement done freely \\
\hline
\end{tabular}

\section{Bibliography}

[1] A. D. Asl and K. Y. Wong, Solving unequal-area static and dynamic facility layout problems using modified particle swarm optimization, J. Intell. Manuf. 28 (2017), 1317-1336.

[2] G. Y. H. Chen, A new data structure of solution representation in hybrid ant colony optimization for large dynamic facility layout problems, Int. J. Product. Econ. 142 (2013), 362-371. 
[3] A. Chraibi, S. Kharraja, I. H. Osman and O. Elbeqqali, A multi-objective mixed-integer programming model for a multisection operating theatre facility layout, in: Proceedings of 2014 International Conference on Operations Research and Enterprise Systems (ICORES), Angers, Loire Valley, France, 2014.

[4] A. Chraibi, S. Kharraja, I. H. Osman and O. Elbeqqali, Optimization of a dynamic operating theatre layout problem, in: Proceedings of 2015 International Conference on Industrial Engineering and Systems Management (IESM), IEEE, Seville, Spain, 2015.

[5] M. Cossentino, C. Lodato, S. Lopes and P. Ribino, Multi agent simulation for decision making in warehouse management, in: 2011 Federated Conference on Computer Science and Information Systems (FedCSIS), pp. 611-618, IEEE, Szczecin, Poland, 2011.

[6] S. Deechongkit and R. Srinon, New mixed integer programming for facility layout design without loss of department area. Ind. Eng. Lett. 4 (2014), 21-27.

[7] Foundation for Intelligent Physical Agents, FIPA ACL Message Structure Specification, Foundation for Intelligent Physical Agents, http://www.fipa.org/specs/fipa00061/SC00061G.html (June 30, 2004), 2002.

[8] I. W. Gibson, in: Proceedings of the 39th Conference on Winter Simulation: 40 years! The best is yet to come, pp. 1501-1509, IEEE Press, Washington, DC, USA, 2007.

[9] H. González-Vélez, M. Mier, M. Julià-Sapé, T. N. Arvanitis, J. M. García-Gómez, M. Robles, P. H. Lewis, S. Dasmahapatra, D. Dupplaw, A. Peet, C. Arús, B. Celda, S. V. Huffe and M. Lluch-Ariet, HealthAgents: distributed multi-agent brain tumor diagnosis and prognosis, Appl. Intell. 30 (2009), 191-202.

[10] A. W. A. Hammad, A. Akbarnezhad and D. Rey, A multi-objective mixed integer nonlinear programming model for construction site layout planning to minimize noise pollution and transport costs, Autom. Construct. 61 (2016), 73-85.

[11] J. Hathhorn, E. Sisikoglu and M. Y. Sir, A multi-objective mixed-integer programming model for a multi-floor facility layout, Int. J. Prod. Res. 51 (2013), 4223-4239.

[12] C. Huang and C. K. Wong, Optimization of site layout planning for multiple construction stages with safety considerations and requirements, Autom. Construct. 53 (2015), 58-68.

[13] D. Isern, D. Sánchez and A. Moreno, Agents applied in health care: a review, Int. J. Med. Inform. 79 (2010), 145-166.

[14] R. Kothari and D. Ghosh, An efficient genetic algorithm for single row facility layout, Optim. Lett. 8 (2014), 679-690.

[15] R. Kothari and D. Ghosh, A scatter search algorithm for the single row facility layout problem, J. Heurist. 20 (2014), $125-142$.

[16] V. G. Koutkias, I. Chouvarda and N. Maglaveras, A multiagent system enhancing home-care health services for chronic disease management, IEEE Trans. Inform. Technol. Biomed. 9 (2005), 528-537.

[17] S. Kulturel-Konak and A. Konak, A large-scale hybrid simulated annealing algorithm for cyclic facility layout problems, Eng. Optim. 47 (2015), 963-978.

[18] N. Lenin, M. Siva Kumar, D. Ravindran and M. N. Islam, A tabu search for multi-objective single row facility layout problem, J. Adv. Manuf. Syst. 13 (2014), 17-40.

[19] Q. L. Lin, H. C. Liu, D. J. Wang and L. Liu, Integrating systematic layout planning with fuzzy constraint theory to design and optimize the facility layout for operating theatre in hospitals, J. Intell. Manuf. 26 (2015), 87-95.

[20] W. E. Lorenz, M. Bicher and G. Wurzer, Adjacency in hospital planning, Math. Modell. 8 (2015), 862-867.

[21] R. Muther, Systematic Layout Planning, no. 658.5 m8, 1973.

[22] N. Nahas and M. Nourelfath, Iterated great deluge for the dynamic facility layout problem, in: Metaheuristics for Production Systems, pp. 57-92, Springer International Publishing, NY, USA, 2016.

[23] H. D. Nataraj Urs, R. C. Biradar, S. S. Manvi, K. M. Sudharshan and K. M. Rudrappa, Floor plan analysis in VLSI design process: multi-agent based approach, in: 2010 International Conference on Communication and Computational Intelligence (INCOCCI), pp. 279-283, IEEE, Erode, India, 2010.

[24] E. H. Nfaoui, O. El Beqqali, Y. Ouzrout and A. Bouras, An approach of decision-making support based on collaborative agents for a large distribution sector, IJISSCM 2 (2008), 16-35.

[25] G. Palubeckis, Fast simulated annealing for single-row equidistant facility layout, Appl. Math. Comput. 263 (2015), $287-301$.

[26] K. S. N. Ripon, K. Glette, K. N. Khan, M. Hovin and J. Torresen, Adaptive variable neighborhood search for solving multiobjective facility layout problems with unequal area facilities, Swarm Evolut. Comput. 8 (2013), 1-12.

[27] M. Shivhare and S. Bansal, Layout optimization in flexible manufacturing system using particle swarm optimization in Matlab, Int. J. Softw. Eng. Appl. 8 (2014), 55-64.

[28] F. G. Tari and H. Neghabi, A new linear adjacency approach for facility layout problem with unequal area departments, J. Manuf. Syst. 37 (2015), 93-103.

[29] H. Tarkesh, A. Atighehchian and A. S. Nookabadi, Facility layout design using virtual multi-agent system, J. Intell. Manuf. 20 (2009), 347-357.

[30] J. A. Tompkins, J. A. White, Y. A. Bozer and J. M. A. Tanchoco, Facilities planning, 4th ed., Wiley, New York, 2010.

[31] M. S. Uddin, Hybrid genetic algorithm and variable neighborhood search for dynamic facility layout problem, Open J. Optim. 4 (2015), 156.

[32] M. Woolridge and N. R. Jennings, Intelligent agents: theory and practice, Knowl. Eng. Rev. 10 (1995), 115-152.

[33] Y. Zhang and A. Che, A mixed integer linear programming approach for a new form of facility layout problem, in: 2014 International Conference on Control, Decision and Information Technologies (CoDIT), pp. 65-68, IEEE, 2014. 